\title{
Existence results for higher order fractional differential equation with multi-point boundary condition
}

Yang Liu

Correspondence: yliu722@163.com Department of Mathematics, Anhui Normal University, Hefei, Anhui, P. R. China

\section{Abstract}

The fixed point theorems on cones are used to investigate the existence of positive solution for higher order fractional differential equation with multi-point boundary condition.

MSC: 26A33; 34B15.

Keywords: fractional differential equation, fixed point, positive solution, cone

\section{Introduction}

Recently, much attention has been paid to the fractional differential equations due to its wide application in physics, engineering, economics, aerodynamics, and polymer rheology etc. For the basic theory and development of the subject, we refer some contributions on fractional calculus, fractional differential equations, see Delbosco [1], Miller [2], and Lakshmikantham et al. [3-7]. Especially, there have been some articles dealing with the existence of solutions or positive solutions of boundary-value problems for nonlinear fractional differential equations (see [8-20] and references along this line). For examples, Jiang [16] obtained the existence of positive solution for boundary value problem of fractional differential equation

$$
D_{0+}^{\alpha} u(t)+f(t, u(t))=0, u(0)=0, u(1)=0,1<\alpha \leq 2,
$$

where $D_{0_{+}}^{\alpha} u(t)$ denotes the standard Riemann-Liouville fractional order derivative. Agarwal et al. [17] investigated the existence of positive solution of singular problem

$$
D_{0+}^{\alpha} u(t)=f\left(t, u(t), D^{\mu} u(t)\right), u(0)=u(1)=0,
$$

where $1<\alpha<2,0 \leq \mu \leq \alpha-1$ and $f$ satisfies the Caratheodory conditions on [0,1] $\times$ $[0, \infty) \times R$ and $f(t, x, y)$ is singular at $x=0$. The existence results of positive solutions are established by using regularization and sequential techniques.

As to the nonlocal problem, Bai [18] established the existence of positive solution for three-point boundary value problem of fractional differential equation

$$
D_{0+}^{\alpha} u(t)+f(t, u(t))=0, u(0)=0, u(1)=\beta u(\eta), \quad \eta \in(0,1) .
$$

By using the fixed point theorems on cones, Li et al. [19] established the existence of positive solutions for problem

(c) 2012 Yang; licensee Springer. This is an open access article distributed under the terms of the Creative Commons Attribution License (http://creativecommons.org/licenses/by/2.0), which permits unrestricted use, distribution, and reproduction in any medium, provided the original work is properly cited. 


$$
D_{0+}^{\alpha} u(t)+f(t, u(t))=0, u(0)=0, \quad D_{0+}^{\beta} u(1)=a D_{0+}^{\beta} u(\xi),
$$

where $1<\alpha \leq 2,0 \leq \beta \leq 1,0 \leq a \leq 1, \xi L(0,1)$ and $a \not 2^{\alpha-\beta-2} \leq 1-\beta, 0 \leq \alpha-\beta-1$ and $f:[0,1] \times[0, \infty) \rightarrow[0, \infty)$ satisfies Caratheodory type conditions.

Very recently, Moustafa and Nieto [20] considered the nontrivial solution for following higher order multi-point problem

$$
\begin{aligned}
& D_{0+}^{\alpha} u(t)+f(t, u(t))=0, n-1 \leq \alpha \leq n, n \in N \\
& u(0)=u^{\prime}(0)=\cdots=u^{(n-2)}=0, \quad u(1)=\sum_{i=1}^{m-2} \beta_{i} u\left(\eta_{i}\right),
\end{aligned}
$$

where $n \geq 2,0<\eta_{i}<1, \beta_{i}>0, i=1,2, \ldots, m-2, \sum_{i=1}^{m-2} \beta_{i} \eta_{i}^{\alpha-1}<1, f \in C([0,1] \times R$, $R)$. The existence of nontrivial solution was established by using the nonlinear alternative of Leray-Schauder. But, existence of positive solution for problem (1.1), (1.2), as far as we know, has not been considered before. Considering that problem (1.1) and (1.2) are more general than problems studied before, we believe that it is interesting to investigate the existence of positive solution for this problem.

In this article, we consider the existence and multiplicity of positive solutions for problem (1.1) and (1.2). We obtain some properties of the associated Green's function. By using these properties of Green's function and fixed point theorems on cones, we establish the existence and multiplicity of positive solutions.

\section{Preliminaries}

For the convenience of the reader, we present here the basic definitions and theory from fractional calculus theory. These definitions and theory can be founded in the literature [1].

Definition 2.1 The fractional integral of order $\alpha>0$ of a function $u(t):(0, \infty) \rightarrow R$ is given by

$$
I_{0+}^{\alpha} u(t)=\frac{1}{\Gamma(\alpha)} \int_{0}^{t}(t-s)^{\alpha-1} u(s) d s
$$

provided the right side is point-wise defined on $(0, \infty)$.

Definition 2.2 The fractional derivative of order $\alpha>0$ of a continuous function $u(t)$ : $(0, \infty) \rightarrow R$ is given by

$$
D_{0+}^{\alpha} u(t)=\frac{1}{\Gamma(n-\alpha)}\left(\frac{d}{d t}\right)^{n} \int_{0}^{t} \frac{u(s)}{(t-s)^{\alpha-n+1}} d s
$$

where $n=[\alpha]+1$, provided that the right side is point-wise defined on $(0, \infty)$.

Lemma 2.1 Let $\alpha>0$. If we assume $u \in C(0,1) \cup L(0,1)$, then problem $D_{0_{+}}^{\alpha} u(t)=0$ has solution

$$
u(t)=C_{1} t^{\alpha-1}+C_{2} t^{\alpha-2}+\cdots+C_{N} t^{\alpha-N}
$$


for some $C_{i} \in R, i=1,2, \ldots, N$, where $N$ is the smallest integer greater than or equal to $\alpha$.

Lemma 2.2 Assume that $u \in C(0,1) \cup L(0,1)$ with a fractional derivative of order $\alpha>$ 0 that belongs to $C(0,1) \cup L(0,1)$. Then

$$
I_{0+}^{\alpha} D_{0+}^{\alpha} u(t)=u(t)+C_{1} t^{\alpha-1}+C_{2} t^{\alpha-2}+\cdots+C_{N} t^{\alpha-N},
$$

for some $C_{i} \in R, i=1,2, \ldots, N$.

Lemma 2.3 [21] Let $E$ be a Banach space and $K \subset E$ be a cone. Assume $\Omega_{1}, \Omega_{2}$ are open bounded subsets of $E$ with $0 \in \Omega_{1} \subset \bar{\Omega}_{1} \subset \Omega_{2}$, and let

$$
A: K \cap\left(\Omega_{2} \backslash \bar{\Omega}_{1}\right) \rightarrow K
$$

be a completely continuous operator such that

$$
\begin{aligned}
& \|A u\| \leq\|u\|, u \in K \cap \partial \Omega_{1}, \text { and }\|A u\| \geq\|u\|, u \in K \cap \partial \Omega_{2} \text { or } \\
& \|A u\| \geq\|u\|, u \in K \cap \partial \Omega_{1}, \text { and }\|A u\| \leq\|u\|, u \in K \cap \partial \Omega_{2},
\end{aligned}
$$

then $A$ has a fixed point in $K \cap\left(\Omega_{2} \backslash \bar{\Omega}_{1}\right)$.

Let $0<a<b$ be given and let $\psi$ be a nonnegative continuous concave functional on the cone $C$. Define the convex sets $C_{r}$ and $C(\psi, a, b)$ by

$$
\begin{gathered}
C_{r}=\{u \in C \mid\|u\|<r\} \\
C(\psi, a, b)=\{u \in C \mid a \leq \psi(u),\|u\| \leq b\} .
\end{gathered}
$$

Lemma 2.4 [22] Let $T: \bar{C}_{r} \rightarrow \bar{C}_{r}$ be a completely continuous operator and let $\psi$ be a nonnegative continuous concave functional on $C$ such that $\psi(u) \leq\|u\|$ for all $u \in \bar{C}_{r}$. Suppose that there exist $0<a<b<d \leq c$ such that

$\left(S_{1}\right)\{u \in C(\psi, b, d) \mid \psi(u)>b\} \neq \varnothing$ and $\psi(T u)>b$ for $u \in C(\psi, b, d)$,

$\left(S_{2}\right)\|T u\|<a$ for $\|u\| \leq a$ and

$\left(S_{3}\right) \psi(T u)>b$ for $u \in C(\psi, b, c)$ with $\|T u\| \geq d$.

Then $T$ has at least three fixed points $u_{1}, u_{2}$, and $u_{3}$ such that

$$
\left\|u_{1}\right\|<a, b<\psi\left(u_{2}\right), \quad\left\|u_{3}\right\|>a, \quad \psi\left(u_{3}\right)<b .
$$

Lemma 2.5 Denote $\eta_{0}=0, \eta_{m-1}=1$ and $\beta_{0}=\beta_{m-1}=0$. Given $y(t) \in C[0,1]$. The problem

$$
D_{0+}^{\alpha} u(t)+\gamma(t)=0, u(0)=u^{\prime}(0)=\cdots=u^{(n-2)}=0, u(1)=\sum_{i=1}^{m-2} \beta_{i} u\left(\eta_{i}\right)
$$

is equivalent to

$$
u(t)=\int_{0}^{1} G(t, s) y(s) d s,
$$

where

$$
G(t, s)=\left\{\begin{array}{l}
\frac{t^{\alpha-1}}{\Gamma(\alpha)\left(1-\sum_{k=0}^{m-1} \beta_{k} \eta_{k}^{\alpha-1}\right)}\left[(1-s)^{\alpha-1}-\sum_{k=i}^{m-1} \beta_{k}\left(\eta_{k}-s\right)^{\alpha-1}\right] t \leq s \\
-\frac{(t-s)^{\alpha-1}}{\Gamma(\alpha)}+\frac{t^{\alpha-1}}{\Gamma(\alpha)\left(1-\sum_{k=0}^{m-1} \beta_{k} \eta_{k}^{\alpha-1}\right)}\left[(1-s)^{\alpha-1}-\sum_{k=i}^{m-1} \beta_{k}\left(\eta_{k}-s\right)^{\alpha-1}\right] t \geq s
\end{array}\right.
$$


Furthermore, the function $G(t, s)$ is continuous on $[0,1] \times[0,1]$ and satisfies the condition

$$
G(t, s)>0, t, s \in[0,1]
$$

Proof. From Lemma 2.1, we get that problem (3.1) is equivalent to

$$
u(t)=-\int_{0}^{t} \frac{(t-s)^{\alpha-1}}{\Gamma(\alpha)} \gamma(s) d s+C_{1} t^{\alpha-1}+C_{2} t^{\alpha-2}+\cdots+C_{n} t^{\alpha-n} .
$$

The boundary conditions $u(0)=u^{\prime}(0)=\ldots=u(n-2)=0$ induce that $C_{2}=C_{3}=\ldots=$ $C_{n}=0$. Considering the boundary condition $u(1)=\sum_{i=0}^{m-1} \beta_{i} u\left(\eta_{i}\right)$, we get

$$
\begin{gathered}
C_{1}=\frac{1}{\left(1-\sum_{i=0}^{m-1} \beta_{i} \eta_{i}^{\alpha-1}\right) \Gamma(\alpha)}\left[\int_{0}^{1}(1-s)^{\alpha-1} \gamma(s) d s-\sum_{i=0}^{m-1} \beta_{i} \int_{0}^{\eta_{i}}\left(\eta_{i}-s\right)^{\alpha-1} \gamma(s) d s\right] . \\
u(t)=-\frac{1}{\Gamma(\alpha)} \int_{0}^{t}(t-s)^{\alpha-1} \gamma(s) d s+\frac{t^{\alpha-1}}{\left(1-\sum_{i=0}^{m-1} \beta_{i} \eta_{i}^{\alpha-1}\right) \Gamma(\alpha)} \\
\times\left[\int_{0}^{1}(1-s)^{\alpha-1} \gamma(s) d s-\sum_{i=0}^{m-1} \beta_{i} \int_{0}^{\eta_{i}}\left(\eta_{i}-s\right)^{\alpha-1} \gamma(s) d s\right] .
\end{gathered}
$$

Then for $\eta_{i-1}<t<\eta_{i}, i=1,2, \ldots, m-1$,

$$
\begin{aligned}
u(t)= & -\frac{1}{\Gamma(\alpha)} \int_{0}^{t}(t-s)^{\alpha-1} \gamma(s) d s \\
& +\frac{t^{\alpha-1}}{\left(1-\sum_{i=1}^{m-2} \beta_{i} \eta_{i}^{\alpha-1}\right) \Gamma(\alpha)}\left[\int_{0}^{1}(1-s)^{\alpha-1} \gamma(s) d s-\sum_{i=1}^{m-2} \beta_{i} \int_{0}^{\eta_{i}}\left(\eta_{i}-s\right)^{\alpha-1} \gamma(s) d s\right] \\
= & \sum_{k=1}^{i-1} \int_{\eta_{k-1}}^{\eta_{k}}\left[-\frac{1}{\Gamma(\alpha)}(t-s)^{\alpha-1}+\frac{t^{\alpha-1}}{\left(1-\sum_{i=1}^{m-2} \beta_{i} \eta_{i}^{\alpha-1}\right) \Gamma(\alpha)}\left((1-s)^{\alpha-1}-\sum_{j=k}^{m-1} \beta_{j}\left(\eta_{j}-s\right)^{\alpha-1}\right)\right] \gamma(s) d s \\
& +\int_{\eta_{i-1}}^{t}\left[-\frac{1}{\Gamma(\alpha)}(t-s)^{\alpha-1}+\frac{t^{\alpha-1}}{\left(1-\sum_{i=1}^{m-2} \beta_{i} \eta_{i}^{\alpha-1}\right) \Gamma(\alpha)}\left((1-s)^{\alpha-1}-\sum_{j=i}^{i-1} \beta_{j}\left(\eta_{j}-s\right)^{\alpha-1}\right)\right] \gamma(s) d s \\
& +\int_{t}^{\eta_{i}} \frac{t^{\alpha-1}}{\left(1-\sum_{i=1}^{m-2} \beta_{i} \eta_{i}^{\alpha-1}\right) \Gamma(\alpha)}\left[(1-s)^{\alpha-1}-\sum_{j=i}^{m-1} \beta_{j}\left(\eta_{j}-s\right)^{\alpha-1}\right] \gamma(s) d s \\
& +\sum_{k=i}^{m-1} \int_{\eta_{k-1}}^{\eta_{k}} \frac{t^{\alpha-1}}{\left(1-\sum_{i=1}^{m-2} \beta_{i} \eta_{i}^{\alpha-1}\right) \Gamma(\alpha)}\left[(1-s)^{\alpha-1}-\sum_{j=k}^{m-1} \beta_{j}\left(\eta_{j}-s\right)^{\alpha-1}\right] \gamma(s) d s=\int_{0}^{1} G(t, s) \gamma(s) d s
\end{aligned}
$$

Furthermore, for $\eta_{i-1} \leq s \leq \eta_{\dot{v}} i=1,2, \ldots, m-1$ and $t \leq s$

$$
\left(\Gamma(\alpha)\left(1-\sum_{k=0}^{m-1} \beta_{k} \eta_{k}^{\alpha-1}\right)\right) G(t, s) \geq t^{\alpha-1} \sum_{k=i}^{m-2}\left((1-s)^{\alpha-1}-\left(\eta_{k}-s\right)^{\alpha-1}\right)>0 .
$$


For $\eta_{i-1} \leq s \leq \eta_{i} i=1,2, \ldots, m-1$ and $t \geq s$

$$
\begin{aligned}
& \left(\Gamma(\alpha)\left(1-\sum_{k=0}^{m-1} \beta_{k} \eta_{k}^{\alpha-1}\right)\right) G(t, s) \geq t^{\alpha-1}\left[(1-s)^{\alpha-1}-\left(1-\frac{s}{t}\right)^{\alpha-1}\right] \\
& +t^{\alpha-1} \sum_{k=0}^{i-1} \beta_{k} \eta_{k}^{\alpha-1}\left[\left(1-\frac{s}{t}\right)^{\alpha-1}-\left(1-\frac{s}{\eta_{k}}\right)^{\alpha-1}\right]>0 .
\end{aligned}
$$

$\square$

Lemma 2.6 The function $G(t, s)$ satisfies the following conditions:

(1) $G(t, s) \leq G(s, s), t, s \in[0,1]$,

(2) There exists function $\gamma(s)$ such that $\min _{\eta_{m-2} \leq s \leq 1} G(t, s) \geq \gamma(s) G(s, s), 0<s<1$.

Proof (1) For $\eta_{i-1}<s<\eta_{i} i=1,2, \ldots, m-1$, Denote

$$
\begin{gathered}
g_{1}(t, s)=\frac{t^{\alpha-1}}{\Gamma(\alpha)\left(1-\sum_{k=0}^{m-1} \beta_{k} \eta_{k}^{\alpha-1}\right)}\left[(1-s)^{\alpha-1}-\sum_{k=i}^{m-1} \beta_{k}\left(\eta_{k}-s\right)^{\alpha-1}\right], \\
g_{2}(t, s)=-\frac{(t-s)^{\alpha-1}}{\Gamma(\alpha)}+\frac{t^{\alpha-1}}{\Gamma(\alpha)\left(1-\sum_{k=0}^{m-1} \beta_{k} \eta_{k}^{\alpha-1}\right)}\left[(1-s)^{\alpha-1}-\sum_{k=i}^{m-1} \beta_{k}\left(\eta_{k}-s\right)^{\alpha-1}\right] .
\end{gathered}
$$

The facts that

$$
\begin{gathered}
\frac{\partial g_{1}(t, s)}{\partial t}=\frac{(\alpha-1) t^{\alpha-2}}{\Gamma(\alpha)\left(1-\sum_{k=0}^{m-1} \beta_{k} \eta_{k}^{\alpha-1}\right)}\left[(1-s)^{\alpha-1}-\sum_{k=i}^{m-1} \beta_{k}\left(\eta_{k}-s\right)^{\alpha-1}\right]>0, \\
\frac{\partial g_{2}(t, s)}{\partial t}=-\frac{(\alpha-1)(t-s)^{\alpha-2}}{\Gamma(\alpha)}+\frac{(\alpha-1) t^{\alpha-2}}{\Gamma(\alpha)\left(1-\sum_{k=0}^{m-1} \beta_{k} \eta_{k}^{\alpha-1}\right)}\left[(1-s)^{\alpha-1}-\sum_{k=i}^{m-1} \beta_{k}\left(\eta_{k}-s\right)^{\alpha-1}\right]<0
\end{gathered}
$$

imply that $g_{1}(t, s)$ is decreasing with respect to $t$ for $\left[\eta_{i-1}, s\right]$ and $g_{2}(t, s)$ is increasing with respect to $t$ for $\left[s, \eta_{i}\right], i=1,2, \ldots, m-1$. Thus one can easily check that

$$
G(t, s) \leq G(s, s), t, s \in[0,1] .
$$

(2) For $\eta_{m-2}<t<1$, denote

$$
\begin{aligned}
& \gamma_{i}(t, s)=-\frac{(t-s)^{\alpha-1}}{\Gamma(\alpha)}+\frac{t^{\alpha-1}}{\Gamma(\alpha)\left(1-\sum_{k=0}^{m-1} \beta_{k} \eta_{k}^{\alpha-1}\right)}\left[(1-s)^{\alpha-1}-\sum_{k=i}^{m-1} \beta_{k}\left(\eta_{k}-s\right)^{\alpha-1}\right], \\
& \gamma(s)=\min \left\{\gamma_{i}\left(\eta_{m-2}, s\right), \frac{1}{\Gamma(\alpha)\left(1-\sum_{k=0}^{m-1} \beta_{k} \eta_{k}^{\alpha-1}\right)}\left[(1-s)^{\alpha-1}-\sum_{k=i}^{m-1} \beta_{k}\left(\eta_{k}-s\right)^{\alpha-1}\right]\right\}, i=1,2, \ldots, m-2 \\
& \gamma_{i}(t, s)=-\frac{(t-s)^{\alpha-1}}{\Gamma(\alpha)}+\frac{t^{\alpha-1}}{\Gamma(\alpha)\left(1-\sum_{k=0}^{m-1} \beta_{k} \eta_{k}^{\alpha-1}\right)}\left[(1-s)^{\alpha-1}-\sum_{k=i}^{m-1} \beta_{k}\left(\eta_{k}-s\right)^{\alpha-1}\right], \\
& \gamma(s)=\min \left\{\gamma_{i}\left(\eta_{m-2}, s\right), \frac{1}{\Gamma(\alpha)\left(1-\sum_{k=0}^{m-1} \beta_{k} \eta_{k}^{\alpha-1}\right)}\left[(1-s)^{\alpha-1}-\sum_{k=i}^{m-1} \beta_{k}\left(\eta_{k}-s\right)^{\alpha-1}\right]\right\}, i=1,2, \ldots, m-2
\end{aligned}
$$

Thus we have

$$
\gamma(s)>0, \min _{\eta_{m-2}<t<1} G(t, s) \geq \gamma(s) G(s, s)=\gamma(s) \max _{0 \leq t \leq 1} G(t, s) .
$$




\section{Main results}

Let $X=C[0,1]$ be a Banach space endowed with the norm

$$
\|u\|=\max _{0 \leq t \leq 1}|u(t)|, u \in X .
$$

Define the cone $P \subset E$ by $P=\{u \in X \mid u(t) \geq 0\}$.

Theorem 3.1 Define the operator $T: P \rightarrow X$,

$$
T u(t)=\int_{0}^{1} G(t, s) f(s, u(s)) d s,
$$

then $T: P \rightarrow P$ is completely continuous.

Proof From the nonnegative and continuous properties of function $f$ and $G(t, s)$, one can obtain easily that the operator $T: P \rightarrow P$ and $T$ is continuous. Let $\Omega$ be a bounded subset of cone $P$. That is, there exists a positive constant $M_{1}>0$ such that $\|u\| \leq M_{1}$ for all $u \in \Omega$. Thus for each $u \in \Omega, t_{1}, t_{2} \in[0,1]$, one has

$$
\begin{aligned}
\left|T u\left(t_{1}\right)-T u\left(t_{2}\right)\right|= & \left|\int_{0}^{1}\left(G\left(t_{1}, s\right)-G\left(t_{2}, s\right)\right) f(s, u(s)) d s\right| \\
& \leq \int_{0}^{1}\left|G\left(t_{1}, s\right)-G\left(t_{2}, s\right)\right| f(s, u(s)) d s \\
& \leq M_{2} \int_{0}^{1}\left|G\left(t_{1}, s\right)-G\left(t_{2}, s\right)\right| d s
\end{aligned}
$$

Then the continuity of function $G(t, s)$ implies that $T$ is equicontinuity on the bounded subset of $P$. On the other hand, for $u \in \Omega$, there exist constant $M_{2}>0$ such that

$$
f(t, u) \leq M_{2}, t \in[0,1], u \in \Omega .
$$

Then

$$
T u(t)=\int_{0}^{1} G(t, s) f(s, u(s)) d s \leq M_{2} \int_{0}^{1} G(s, s) d s .
$$

which implies that $T$ is uniformly bounded on the bounded subset of $P$. Then an application of Ascoli-Arezela ensures that $T: P \rightarrow P$ is completely continuous.

Theorem 3.2 Assume that there exist two positive constant $r_{2}>\frac{N}{M} r_{1}>0$ such that (A1) $f(t, u) \leq M r_{2},(t, u) \in[0,1] \times\left[0, r_{2}\right]$

(A2) $f(t, u) \geq N r_{1},(t, u) \in[0,1] \times\left[0, r_{1}\right]$

where

$$
M=\left(\int_{0}^{1} G(s, s) d s\right)^{-1}, N=\left(\int_{V_{m-2}}^{1} \gamma(s) G(s, s) d s\right)^{-1}
$$


Then problem (1.1) and (1.2) has at least one positive solution $u$ such that $r_{1} \leq\|u\|$ $\leq r_{2}$.

Proof Let $\Omega_{2}=\left\{u \in P \mid\|u\| \leq r_{2}\right\}$. For $u \in \partial \Omega_{2}$, considering assumption (A1), we have

$$
\begin{aligned}
0 & \leq u(t) \leq r_{1}, \text { and } f(t, u) \geq M r_{2}, t \in[0,1], \\
T u(t) & =\int_{0}^{1} G(t, s) f(s, u(s)) d s \leq M r_{2} \int_{0}^{1} G(s, s) d s \leq r_{2} .
\end{aligned}
$$

Thus $\|T u\| \leq\|u\|, u \in \partial \Omega_{2}$.

Let $\Omega_{1}=\left\{u \in P \mid\|u\| \leq r_{1}\right\}$. For $u \in \partial \Omega_{1}$, considering assumption (A2), we have

$$
0 \leq u(t) \leq r_{1} \text {, and } f(t, u) \geq N r_{2}, t \in[0,1]
$$

Thus for $t \in\left[\eta_{m-2}, 1\right]$, we get

$$
T u(t)=\int_{0}^{1} G(t, s) f(s, u(s)) d s \geq \int_{\eta_{m-2}}^{1} \gamma(s) G(s, s) f(s, u(s)) d s \geq r_{1},
$$

which gives that $\|T u\| \geq\|u\|, u \in \partial \Omega_{1}$. An application of Lemma (2.5) ensures the existence of positive solution $u(t)$ of problem (1.1) and (1.2).

Theorem 3.3 Suppose that there exist constants $0<a<b<c$ such that

(A3) $f(t, u)<M a$, for $(t, u) \in[0,1] \times[0, a]$,

(A4) $f(t, u) \geq N b$, for $(t, u) \in\left[\eta_{m-2}, 1\right] \times[b, c]$,

(A5) $f(t, u) \leq M c$, for $(t, u) \in[0,1] \times[0, c]$,

then problem (1.1) and (1.2) has at least three positive solution $u_{1}, u_{2}, u_{3}$ with

$$
\max _{0 \leq t \leq 1}\left|u_{1}\right| \leq a, b<\min _{\eta_{m-2} \leq t \leq 1}\left|u_{2}\right|<\max _{0 \leq t \leq 1}\left|u_{2}\right| \leq c, a<\max _{0 \leq t \leq 1}\left|u_{3}\right| \leq c, \min _{\eta_{m-2} \leq t \leq 1}\left|u_{3}\right|<b .
$$

Proof Let the nonnegative continuous concave functional $\theta$ on the cone $P$ defined by

$$
\theta(u)=\min _{\eta_{m-2} \leq t \leq 1}|u(t)| .
$$

If $u \in \bar{P}_{c}$, then $\|u\| \leq c$. Then by condition (A5), we have

$$
f(t, u) \leq M c, \text { for }(t, u) \in[0,1] \times[0, c] .
$$

Thus

$$
|T(u)(t)|=\left|\int_{0}^{1} G(t, s) f(s, u(s)) d s\right| \leq M c \int_{0}^{1} G(s, s) d s=c .
$$

which yields that $T: \bar{P}_{c} \rightarrow \bar{P}_{c}$. In the same way, we get that

$$
\|T u\|<a, \text { for } u \leq a \text {. }
$$

We chose the function $u(t)=\frac{b+c}{2} t \in[0,1]$. We claim that $\frac{b+c}{2} \in\{u \in P(\theta, b, c) \mid \theta(u)>b\}$, which ensures that $\{u \in P(\theta, b, c) \mid \theta(u)>b\} \neq \varnothing$. 
And for $u \in P(\theta, b, c)$, we have

$$
f(t, u(t)) \geq N b, t \in\left[\eta_{m-2}, 1\right]
$$

Then

$$
\theta(T u)=\min _{\eta_{m-2} \leq t \leq 1}\left|\int_{0}^{1} G(t, s) f(s, u(s)) d s\right|>N b \int_{\eta_{m-2}}^{1} \gamma(s) G(s, s) d s=b,
$$

which yields that $\theta(T u)>b$, for $u \in P(\theta, b, c)$.

An application of Lemma (2.6) ensures that problem (1.1) and (1.2) has at least three positive solutions with

$$
\max _{0 \leq t \leq 1}\left|u_{1}\right| \leq a, b<\min _{\eta_{m-2} \leq t \leq 1}\left|u_{2}\right|<\max _{0 \leq t \leq 1}\left|u_{2}\right| \leq c, a<\max _{0 \leq t \leq 1}\left|u_{3}\right| \leq c, \min _{\eta_{m-2} \leq t \leq 1}\left|u_{3}\right|<b
$$

\section{Acknowledgements}

This study was supported by the Anhui Provincial Natural Science Foundation (10040606Q50) and the Natural Science Foundation of Anhui Department of Education (KJ2010A285).

\section{Authors' contributions}

The authors declare that the work was realized in collaboration with the same responsibility. All authors read and approved the final manuscript.

\section{Competing interests}

The authors declare that they have no competing interests.

Received: 23 October 2011 Accepted: 15 May 2012 Published: 15 May 2012

\section{References}

1. Delbosco, D: Fractional calculus and function spaces. J Fract Calc [J]. 6, 45-53 (1994)

2. Miller, KS, Ross, B: An Introduction to the Fractional calculus and Fractional Differential Equations. Wiley, New York (1993) Lakshmikantham (Author), S.Leela (Author), J.Vasundhara Devi (Author)

3. Leela, S, Devi, J: Theory of Fractional Dynamic Systems. Cambridge Academic, Cambridge (2009)

4. Lakshmikantham, V, Devi, J: Theory of fractional differential equations in a Banach space. Eur J Pure Appl Math. 1, 38-45 (2008)

5. Lakshmikantham, $V$, Leela, S: Nagumo-type uniqueness result for fractional differential equations. Nonlinear Anal TMA. 71, 2886-2889 (2009). doi:10.1016/j.na.2009.01.169

6. Lakshmikantham, V, Leela, S: A Krasnoselskii-Krein-type uniqueness result for fractional differential equations. Nonlinear Anal TMA. 71, 3421-3424 (2009). doi:10.1016/j.na.2009.02.008

7. Lakshmikantham, V: Theory of fractional differential equations. Nonlinear Anal TMA. 69, 3337-3343 (2008). doi:10.1016/j. na.2007.09.025

8. Zhang, S: The existence of a positive solution for a nonlinear fractional differential equation. J Math Anal Appl. 252, 804-812 (2000). doi:10.1006/jmaa.2000.7123

9. Zhang, S: Existence of positive solution for some class of nonlinear fractional differential equation. J Math Anal Appl. 278, 136-148 (2003). doi:10.1016/50022-247X(02)00583-8

10. Babakhani, A, Gejji, VD: Existence and positive solutions of nonlinear Fractional differential equations. J Math Anal Appl. 278, 434-442 (2003). doi:10.1016/50022-247X(02)00716-3

11. Xu, XJ, Jiang, DQ, Yuan, CJ: Multiple positive solutions for the boundary value problem of a nonlinear fractional differential equations. Nonlinear Anal TMA. 71, 4676-4688 (2009). doi:10.1016/j.na.2009.03.030

12. Liang, S, Zhang, J: Positive solutions for boundary value problems of nonlinear fractional differential equation. Nonlinear Anal. 71, 5545-5550 (2009). doi:10.1016/i.na.2009.04.045

13. Ahmad, B, Nieto, J: Existence results for a coupled system of nonlinear fractional differential equations with three-point boundary conditions. Comput Math Appl. 58, 1838-1843 (2009). doi:10.1016/j.camwa.2009.07.091

14. Bai, Z, Lü, H: Positive solutions for boundary value problem of nonlinear fractional differential equation. J Math Anal Appl [J]. 311, 495-505 (2005)

15. Kosmatov, N: A singular boundary value problem for nonlinear differential equations of fractional order. J Appl Math Comput. 29, 125-135 (2010)

16. Jiang, D, Yuan, C: The positive properties of the Green function for Direchlet-type boundary value problems of nonlinear fractional differential equations and its application. Nonlinear Anal. 15, 710-719 (2010)

17. Agarwal, RP, O'Regan, D, Stanêk, Svatoslav: Positive solutions for Dirichlet problems of sigular nonlinear fractional differential equations. J Math Anal Appl. 371, 57-68 (2010). doi:10.1016/j.jmaa.2010.04.034

18. Bai, Z: On positive solutions of a nonlocal fractional boundary value problem. Nonlinear Anal TMA [J]. 72, 916-924 (2010). doi:10.1016/j.na.2009.07.033 
19. Li, C, Luo, X, Zhou, Y: Existence of positive solutions of the boundary value problem for nonlinear fractional differential equations. Comput Math Appl. 59, 1363-1375 (2010). doi:10.1016/j.camwa.2009.06.029

20. Shahed, M, Nieto, J: Nontrivial solution for a nonlinear multi-point boundary value problem of fractional order. Comput Math Appl. 59, 3438-3443 (2010). doi:10.1016/j.camwa.2010.03.031

21. Krasnosel'skii, M: Topological methods in the theory of nonlinear integral equations. Pergamon, Elmsford (1964)

22. Leggett, R, Williams, L: Multiple positive fixed points of nonlinear operators on ordered Banach spaces. Indiana Univ Math J. 28, 673-688 (1979). doi:10.1512/iumj.1979.28.28046

doi:10.1186/1687-2770-2012-57

Cite this article as: Liu: Existence results for higher order fractional differential equation with multi-point

boundary condition. Boundary Value Problems 2012 2012:57.

\section{Submit your manuscript to a SpringerOpen ${ }^{\circ}$} journal and benefit from:

- Convenient online submission

- Rigorous peer review

- Immediate publication on acceptance

- Open access: articles freely available online

- High visibility within the field

- Retaining the copyright to your article

Submit your next manuscript at $\gg$ springeropen.com 